\title{
Reflets
}

Revue ontaroise d'intervention sociale et communautaire

\section{Services en santé mentale pour les personnes ayant un handicap intellectuel : combler des lacunes}

\section{Robert Leclerc}

Volume 6, numéro 1, printemps 2000

Approches d'intervention : définir et renouveler nos pratiques

URI : https://id.erudit.org/iderudit/026296ar

DOI : https://doi.org/10.7202/026296ar

Aller au sommaire du numéro

Éditeur(s)

Reflets : Revue ontaroise d'intervention sociale et communautaire

ISSN

1203-4576 (imprimé)

1712-8498 (numérique)

Découvrir la revue

Citer cet article

Leclerc, R. (2000). Services en santé mentale pour les personnes ayant un handicap intellectuel : combler des lacunes. Reflets, 6(1), 90-106.

https://doi.org/10.7202/026296ar

Tous droits réservés (C) Reflets : Revue ontaroise d'intervention sociale et communautaire, 2000
Ce document est protégé par la loi sur le droit d'auteur. L'utilisation des services d'Érudit (y compris la reproduction) est assujettie à sa politique d'utilisation que vous pouvez consulter en ligne.

https://apropos.erudit.org/fr/usagers/politique-dutilisation/ 


\title{
Services en santé mentale pour les personnes ayant un handicap intellectuel : combler des lacunes
}

\author{
Robert Leclerc, École de psychologie, \\ Université d'Ottawa
}

\section{Introduction}

Les services en santé mentale sont généralement faciles d'accès, soit par l'entremise d'organismes bien identifiés, comme les centres de santé communautaire, les cliniques spécialisées ou les hôpitaux psychiatriques, soit par le recours à des professionnels en cabinet privé de consultation. L'aide est disponible pour une personne souffrant de dépression sévère ou encore d'anxiété. Toutefois, la situation est différente pour une personne ayant un handicap intellectuel. Si elle souffre d'un problème de santé mentale, les chances qu'elle reçoive de l'aide clinique sont minces, voire même inexistantes (Tanguay et Szymanski 1980). De fait, les personnes ayant un handicap intellectuel constituent, dans notre société, le groupe le moins bien desservi en matière de soins en santé mentale (Reiss, Levitan et McNally 1982).

L'expression «handicap intellectuel» ne correspond pas à une condition médicale ou à un trouble psychologique (Luckasson et al. 1992) ${ }^{1}$. Elle décrit, chez une personne, un état particulier de fonctionnement, débutant à l'enfance, qui est caractérisé par la présence de limites importantes au plan intellectuel, lesquelles sont associées à des déficits importants au niveau des habiletés de 
"...il y a lieu de croire que la fréquence relative de troubles en santé mentale serait plus élevée chez les personnes ayant un handicap intellectuel...» la vie de tous les jours. Par conséquent, l'expression «double diagnostic» signale, chez une même personne, la présence d'un handicap intellectuel et d'un trouble en santé mentale, sans toutefois préciser la nature de celui-ci (Borthwick-Duffy et Eyman 1990; Matson et Barrett 1993; Reiss 1993).

Ainsi, on estime que les personnes ayant un handicap intellectuel pourraient avoir les mêmes problèmes psychologiques que les personnes n'ayant pas de handicap intellectuel (Nezu, Nezu et Gill-Weiss 1992; Reiss 1994) et qu'elles pourraient éprouver toutes les formes des troubles affectifs connus (Pawlarcyzk et Beckwith 1987). Cependant, il y a lieu de croire que la fréquence relative de troubles en santé mentale serait plus élevée chez les personnes ayant un handicap intellectuel (Jacobson 1990) et que ces différences seraient associées à divers facteurs, notamment l'âge de la personne et le niveau de sévérité de son handicap intellectuel (Reiss et Valenti-Hein 1994).

Bien que le manque de services adéquats en santé mentale pour les personnes ayant un double diagnostic ait été dénoncé depuis plus de 20 ans, le problème demeure encore très actuel (Nezu et Nezu 1994). Cette situation est d'autant plus préoccupante que les personnes ayant un handicap intellectuel sont plus fragiles au plan émotionnel que celles n'ayant pas ce type de handicap (Bruininks, Hill et Morreau 1988; Matson et Barrett 1993; Matson et Sevin 1994; Reiss, Levitan et McNally 1982; Singh, Sood, Sonenklar et Ellis 1991). Elles ont donc une plus grande prédisposition à développer des troubles psycho logiques comme des troubles affectifs (Sovner et Pary 1993) ou de personnalité (Reiss 1990). Souvent, leurs limites sont très importantes aux plans de la communication et des habiletés sociales. De plus, leurs capacités cognitives à solutionner des problèmes personnels ou interpersonnels les rendent plus vulnérables au plan émotionnel (Gardner et Moffat 1990).

Dans cet article, nous présenterons et analyserons les différents facteurs liés à la pénurie de services cliniques adéquats pour ces personnes, en faisant appel aux données scientifiques sur le sujet et à notre expérience clinique auprès de francophones ayant un double diagnostic et vivant en Ontario, plus précisément dans la 
région d'Ottawa. Ce petit groupe constitue en quelque sorte une minorité au sein du groupe déjà minoritaire des personnes ayant un double diagnostic. Ces personnes ont donc très peu de poids politique, malgré l'importance de leurs besoins. C'est pourquoi la présente analyse considérera des actions à différents niveaux afin d'y répondre.

\section{Facteurs liés à la pénurie et à l'inadéquation des services}

Parmi les principaux facteurs expliquant le manque de services auprès de cette clientèle, on retrouve :

- la dissimulation diagnostique, qui correspond à la tendance à donner moins d'importance aux signes cliniques de troubles en santé mentale si la personne est préalablement identifiée comme ayant un handicap intellectuel;

- le manque de rigueur dans le processus d'évaluation du handicap intellectuel;

- le manque d'outils diagnostiques adaptés aux personnes ayant un handicap intellectuel et,

- le manque d'intégration des ressources provenant, d'une part, du système des services de santé et, d'autre part, du système des services communautaires et sociaux, problème qui reflète souvent un conflit de valeurs et de philosophies propres à chacun.

Dans la suite du texte, nous développerons chacun de ces aspects de façon plus détaillée.

\section{La dissimulation diagnostique}

La dissimulation diagnostique (diagnostic overshadowing) est l'expression qui permet de décrire ce qui apparaît essentiellement comme une erreur de jugement de la part de cliniciens à qui on a demandé, en se basant sur des études de cas, d'émettre une opinion professionnelle quant aux signes de troubles émotionnels 
«...le fait d'identifier

un handicap

intellectuel chez une

personne aurait comme effet de camoufler chez elle les signes de problèmes de santé mentale». chez des personnes ayant un handicap intellectuel (Reiss, Levitan et Szyszko 1982; Reiss et Szyszko 1983). Par exemple, dans une étude, Reiss et ses collaborateurs (1982) avaient demandé à des psychologues cliniciens de donner leurs impressions diagnostiques sur certaines personnes ayant un handicap intellectuel et d'autres qui n'en avaient pas. Tous ces individus présentaient des signes de troubles émotionnels semblables. Les résultats ont montré que les cliniciens formulaient correctement un diagnostic de troubles émotionnels lorsque les personnes n'avaient pas de handicap intellectuel, mais qu'ils ne formulaient aucun diagnostic de troubles émotionnels ou qu'ils jugeaient celui-ci comme étant moins sévère si les personnes avaient un handicap intellectuel. Ainsi, le fait d'identifier un handicap intellectuel chez une personne aurait comme effet de camoufler chez elle les signes de problèmes de santé mentale.

Diverses explications au phénomène de la dissimulation diagnostique ont été offertes ${ }^{2}$. Par exemple, MacLean (1993) souligne que l'erreur de jugement serait possiblement attribuable à l'influence d'une vision "développementale» de la personne ayant un handicap intellectuel qui consiste à interpréter les comportements de la personne en fonction d'une équivalence d'âge mental, et non de son âge réel. Par exemple, l'agitation motrice chez l'adulte ayant un handicap intellectuel serait interprétée selon des normes de comportement attribuables à un enfant et non, comme le signe d'un état anxieux.

Une autre explication serait liée à la difficulté de séparer les signes du handicap intellectuel (p. ex. l'incohérence langagière associée à des limites au plan de la communication expressive) de certains signes associés à des troubles psychologiques (p. ex. une désorganisation du langage associée à un désordre de la pensée). Dans l'un et l'autre cas, l'évaluateur a tendance à interpréter les agissements problématiques de la personne, non pas comme des signes de troubles psychologiques, mais plutôt comme ceux d'un fonctionnement «normal» chez une personne ayant un handicap intellectuel et, bien sûr, il ne sonnera pas l'alarme.

MacLean (1993) souligne l'importance de l'expérience clinique de la personne qui formule le diagnostic dans des 
«Selon mes

observations, les

francophones de

l'Ontario ayant un

handicap intellectuel

ont souvent été évalués

au plan psychologique

par un psychiatre ne

parlant pas le français

et ne connaissant pas

ou peu les valeurs

culturelles de ce

groupe». situations potentielles de dissimulation diagnostique. En effet, plus grande est l'expérience dans la formulation d'un diagnostic donné, moins grandes sont les chances qu'elle montre une telle erreur de jugement clinique. Cela dit, il est évident que d'autres recherches sont nécessaires pour mieux cerner les variables associées au problème de la dissimulation diagnostique. Une des variables qui a peu fait l'objet d'études à ce jour, est celle des différences culturelles, avec lesquelles sont associées non seulement des habitudes de vie particulières, mais aussi différents styles d'interactions sociales et même, différentes façons de se percevoir et de percevoir les autres (Cheng 1987). Il est particulièrement important de comprendre l'effet des différences culturelles, surtout lorsque l'évaluateur fait partie d'un groupe socioculturel différent de celui de la personne ayant un handicap intellectuel (Lim et Browder 1994). Cette situation représente malheureusement très souvent la réalité des francophones ayant un double diagnostic et vivant en Ontario. Selon mes observations, les francophones de l'Ontario ayant un handicap intellectuel ont souvent été évalués au plan psychologique par un psychiatre ne parlant pas le français et ne connaissant pas ou peu les valeurs culturelles de ce groupe. Typiquement dans ces situations, le psychiatre anglophone communique par l'intermédiaire d'une tierce personne, agissant comme interprète et n'ayant aucune qualification en évaluation psychologique. Ainsi, l'information transmise est interprétée par le psychiatre selon son cadre de référence culturel, ce qui peut résulter en un jugement clinique incomplet ou même erroné.

La dissimulation diagnostique a pour conséquence, le refus de reconnaitre les besoins en santé mentale des personnes ayant un handicap intellectuel, d'où l'absence de services cliniques répondant à leurs problèmes spécifiques. Dans ce contexte, une façon de prévenir le problème de la dissimulation diagnostique serait de suivre une démarche rigoureuse au moment de l'évaluation du handicap intellectuel. Malheureusement, comme il sera possible de le constater dans la section suivante, l'évaluation du handicap intellectuel est souvent faite avec trop peu de rigueur scientifique. 


\section{Le manque de rigueur dans le processus d'évaluation du handicap intellectuel}

Il est difficile de préciser clairement le nombre de personnes ayant un handicap intellectuel et un problème de santé mentale. Rojahn et Tassé (1996) ont fait une analyse comparée de six études, publiées depuis 1980, qui se sont penchées sur cette question et rapportent un pourcentage de personnes ayant un double diagnostic variant entre $10 \%$ et $40 \%$. Selon eux, cette variabilité des résultats serait attribuable aux différences quant aux populations étudiées, à la nature des troubles identifiés, aux instruments diagnostiques utilisés et même à la façon de définir le handicap intellectuel. Pour mieux comprendre les difficultés inhérentes à cette question, il importe d'aborder la question de l'évaluation du handicap intellectuel et les problèmes qui y sont associés.

\section{Définition et processus d'évaluation}

La définition de handicap intellectuel correspond, en termes cliniques, à celle du retard mental ou intellectuel (American Psychiatric Association 1994). En tant que trouble associé au développement de l'enfant, la catégorie diagnostique de la déficience mentale doit obligatoirement inclure les trois critères suivants :

- un déficit significatif au plan cognitif (établi à partir des résultats d'un test de quotient intellectuel valide);

- des déficits significatifs concomitants dans au moins deux domaines de comportements dits adaptatifs ou fonctionnels (mesurés objectivement à l'aide d'échelles standardisées de comportements spécifiques de la vie de tous les jours), et

- l'apparition de tels déficits avant l'âge de 18 ans (ce qui est déterminé à partir de la cueillette d'information sur l'histoire du développement de la personne).

Ces critères suggèrent donc que le diagnostic de la déficience mentale nécessite une documentation très précise, l'administration de tests et l'interprétation adéquate des résultats de ces tests. 
"...mes observations des pratiques courantes m'amènent à constater que...le diagnostic de handicap intellectuel est établi, occasionnellement, sans aucune documentation clinique ou, fréquemment, avec une documentation incomplète».
Il va de soi qu'un diagnostic fiable du handicap intellectuel constitue la première étape dans un processus visant l'identification des troubles de santé mentale chez ces personnes. Dans le contexte de cette première étape, l'information recueillie permet souvent d'anticiper certains problèmes de fonctionnement personnel ou social (Das et Naglieri 1996). Lorsque c'est le cas, le rapport psychologique contient alors des recommandations de suivi diagnostique et même un cadre d'intervention. Malgré l'importance d'établir correctement la présence d'un handicap intellectuel, mes observations des pratiques courantes m'amènent à constater que, encore aujourd'hui, le diagnostic de handicap intellectuel est établi, occasionnellement, sans aucune documentation clinique ou, fréquemment, avec une documentation incomplète. En particulier, l'information récente sur le niveau de fonctionnement adaptatif de la personne (voir le deuxième critère, plus haut) manque le plus souvent. Malheureusement, le manque de rigueur dans l'administration d'une démarche diagnostique objective et complète peut amener la personne en charge d'établir le diagnostic à s'en remettre à des impressions globales et à des sources d'informations peu fiables, ce qui augmente les chances de se tromper. De plus, ce manque de rigueur expose l'évaluateur à des formes implicites de manipulation et de pression. Par exemple, le besoin immédiat d'une ressource particulière (comme le logement ou une source de revenu additionnelle) peut influencer le jugement clinique.

\section{Comment expliquer le manque de rigueur dans le processus d'évaluation?}

Le manque de connaissances chez les intervenants dans le domaine des services de santé en constitue une cause principale. Cette méconnaissance porte sur les critères d'évaluation du handicap intellectuel et les outils d'évaluation qui s'y rattachent et aussi sur les développements conceptuels associés au domaine du handicap intellectuel. Par exemple, la définition de handicap intellectuel (voir plus haut) comporte une dose d'optimisme et d'espoir, en ce sens qu'elle implique l'attente d'amélioration sur le plan du fonctionnement dit adaptatif des personnes ayant un handicap 
"...les intervenants

dans le domaine des

services de santé

doivent avoir plus de

formation relative aux

réalités sociales et

psychologiques propres

au domaine $d u$

handicap intellectuel». intellectuel. De fait, elle suggère que le handicap intellectuel ne doive plus être considéré comme une condition permanente chez la personne. Il peut donc disparaître, mais aussi réapparaitre, selon les exigences de l'environnement et le support présent dans la communauté 3 . Il en découle que les intervenants dans le domaine des services de santé doivent avoir plus de formation relative aux réalités sociales et psychologiques propres au domaine du handicap intellectuel.

Un autre facteur pouvant contribuer à ce manque de rigueur est le souci des personnes et des conséquences possibles liées aux résultats de l'évaluation. Par exemple, les adultes qui reçoivent présentement divers services (scolaires, sociaux ou communautaires) pourraient ne plus en bénéficier, si les résultats démontraient qu'elles n'avaient pas un handicap intellectuel. De même, un résultat semblable pourrait empêcher les personnes qui font une demande de services de les obtenir. Une telle possibilité pourrait se matérialiser dans les cas où le niveau de fonctionnement cognitif serait significativement supérieur à un niveau de quotient intellectuel (QI) global de 70 ou 75 (Smith 1994). Cette inquiétude est légitime.

De fait, tout devrait être mis en place pour répondre aux besoins particuliers de ceux et celles qui n'ont pas de handicap intellectuel, mais qui auraient possiblement des troubles d'apprentissage. Il est clair que ces personnes font face, elles aussi, à une pénurie de services adéquats. Dans ce contexte, le fait d'être confondu aux personnes ayant un handicap intellectuel devient une façon de combler l'absence de services. Bien qu'il soit difficile d'en évaluer l'importance, une telle tendance existe. Toutefois, il importe de la contrer. Il est donc raisonnable de vouloir distinguer clairement entre les personnes qui ont de graves limites de fonctionnement (handicap intellectuel) et celles qui n'en ont pas (par exemple, trouble d'apprentissage), de façon à donner à chacun des services qui leur conviennent.

Pour ce faire, il semble justifiable et raisonnable de vouloir bien identifier les personnes ayant un handicap intellectuel, à partir d'une démarche diagnostique éprouvée, pour ensuite pouvoir identifier leurs besoins en santé mentale (Borthwick-Duffy et Eyman 1990). 
Toutefois, cette démarche clinique ne fait pas l'unanimité parmi les gens qui oeuvrent auprès des personnes qui ont un handicap intellectuel, parce qu'ils craignent, en bonne partie, toute forme d'étiquetage pouvant conduire à diverses pratiques de stigmatisation (Langer et Abelson 1974; Weisz 1981). Cela dit, une démarche diagnostique rigoureuse est nécessaire au développement de stratégies thérapeutiques efficaces. De plus, le processus d'évaluation qui débute avec l'évaluation du handicap intellectuel est utile, non seulement pour des considérations cliniques, mais aussi pour des considérations éducatives. Ce processus d'évaluation devrait se poursuivre tout au long de la vie de la personne de façon à pouvoir démontrer que les programmes scolaires, sociaux et communautaires répondent réellement aux besoins des individus et qu'ils produisent des gains concrets au niveau du développement d'habiletés fonctionnelles pertinentes aux personnes ayant un handicap intellectuel (Baine 1996).

\section{Les lacunes aux plans des outils diagnostiques}

Les outils diagnostiques en santé mentale ont été développés pour la population en général et ne sont donc pas adaptés aux personnes ayant un handicap intellectuel, parce qu'ils ne tiennent pas compte de leurs limites de fonctionnement. Par exemple, les tests diagnostiques et les interviews cliniques nécessitent, de la part de

"Le développement

d'instruments

diagnostiques mieux

adaptés aux personnes

ayant un handicap

intellectuel a connu un

essor considérable au

cours des dix dernières

années...» la personne évaluée, des capacités relativement normales au plan de la compréhension verbale et au plan de l'expression verbale. Or, ces capacités sont typiquement limitées, voire très limitées ou même absentes, chez les personnes qui ont un handicap intellectuel, d'où la nécessité de développer de nouveaux instruments diagnostiques (Pawlarcyzk et Beckwith 1987).

Le développement d'instruments diagnostiques mieux adaptés aux personnes ayant un handicap intellectuel a connu un essor considérable au cours des dix dernières années et ceux qui sont maintenant disponibles visent à évaluer soit l'ensemble des troubles psychologiques, soit des problèmes spécifiques, chez ces personnes ${ }^{4}$. De plus, les recherches en cours visent à en améliorer la valeur clinique et la valeur psychométrique, qualités qui étaient généralement faibles chez les premiers instruments utilisés pour 
établir un double diagnostic (Sturmey, Reed et Corbett 1991). Par exemple, de récentes recherches tentent de mieux identifier, chez les personnes qui ont un handicap intellectuel, la présence d'états dépressifs (Matson, Rush, Hamilton, Anderson, Bamburg et Baglio 1999) et la possibilité de comportements suicidaires (Hardan et Sahl 1999). D'autres recherches visent à évaluer la validité ou la fiabilité soit des instruments diagnostiques le plus souvent utilisés (Matson, Bamburg, Cherry et Paclawskyj 1999; Walsh et Shenouda 1999), soit de stratégies de mesure visant à identifier la fonction des troubles de comportement comme l'automutilation et l'agressivité (Martin, Gaffan et Williams 1999).

À cause de la complexité des problématiques chez les personnes ayant un double diagnostic, il est souhaitable d'avoir une approche multidisciplinaire, donc une approche qui fait appel à plusieurs sources d'information (Dosën 1993). Une telle approche a été mise de l'avant pour mieux cerner, chez les personnes ayant un double diagnostic, les causes des comportements agressifs envers les autres (Garner et Cole 1993). Ce problème, à lui seul, monopolise les quelques ressources d'aide dans la communauté et constitue un obstacle majeur à l'intégration sociale en raison de son association à des actes criminels (Baroff 1996). Dans le cas des gestes agressifs, ceux-ci pourraient signifier soit un trouble de perception (p. ex. psychotique), soit une stratégie apprise de contrôle de l'environnement, soit une erreur d'analyse cognitive. Ces différents diagnostics donneraient alors lieu à des interventions différentes, soit de nature psycho-pharmacologique, soit behavioriste (Van Houten et Axelrod 1993), soit de restructuration cognitive (Black, Cullen et Novaco 1997) ou une combinaison de ces interventions.

Les résultats de recherche à venir nous permettront sûrement "...même armés d'outils puissants, nous ne pouvons pas espérer régler le problème du manque de services cliniques auprès des personnes qui ont un double diagnostic..." de développer des instruments diagnostiques valables et utiles et il sera alors possible de mieux cibler un traitement précis qui apportera une aide efficace aux personnes ayant un double diagnostic. Il est clair qu'il reste beaucoup à faire au niveau des recherches cliniques. Pourtant, même armés d'outils puissants, nous ne pouvons pas espérer régler le problème du manque de services cliniques auprès des personnes qui ont un double diagnostic sans 
qu'il y ait en même temps une cohésion dans l'action des intervenants des services de santé, d'une part, et des intervenants des services sociaux, d'autre part.

\section{Manque d'intégration des ressources des systèmes de services de santé, communautaires et sociaux}

«...ce sont les

personnes qui ont un double diagnostic qui en font les frais, puisqu'elles ne reçoivent pas les services dont elles ont besoin».
Le processus de désinstitutionnalisation a permis à des milliers de personnes ayant un handicap intellectuel d'accéder à des conditions de vie normale. Pour faciliter leur intégration au sein de la communauté, divers services ont été créés répondant à des besoins immédiats comme le logement ou à des besoins à plus long terme comme l'intégration dans le milieu de travail. En Ontario, ce processus a débuté vers le milieu des années 1970, parallèlement à un transfert de responsabilités (et de budgets) du ministère de la Santé vers le ministère des Services sociaux et communautaires. Aujourd'hui, donc 30 ans plus tard, ces services sont nombreux et diversifiés, mais ils manquent de coordination et de cohésion. Il n'y a que peu ou pas d'interaction entre les services sociaux et communautaires et les services de soins en santé mentale. Malheureusement, ce sont les personnes qui ont un double diagnostic qui en font les frais, puisqu'elles ne reçoivent pas les services dont elles ont besoin. En effet, chacun de ces ministères se renvoie la balle en ce qui concerne le manque d'intégration des ressources. Bien sûr, cette situation n'est pas particulière à l'Ontario, car elle est observable ailleurs, notamment aux ÉtatsUnis (Woodward 1993). Woodward (1993) propose certaines explications au problème du manque d'intégration des services de santé et des services communautaires.

D'abord, il note que les différences au niveau des énoncés de mission et des politiques des services reflètent des différences profondes au niveau de la formation reçue (et donc des valeurs). Il existe donc des points de vue divergents qui s'expriment souvent par l'emploi de mots ou d'expressions différentes pour désigner la même personne. Ensuite, la réalité même du double diagnostic crée un doute quant au système de services qui devrait en être saisi. En effet, la personne qui a un double diagnostic a des 
«...pour répondre au problème du cloisonnement professionnel et au problème d'attitude défavorable quant aux réalités associées au double diagnostic, il serait utile de mettre sur pied un programme conjoint de formation offert aux intervenants en santé et à ceux des services sociaux et communautaires». difficultés qui la rendent peu désirables, ce qui fait que l'un et l'autre des systèmes de services en refusent la responsabilité. Finalement, les façons habituelles de faire dans l'un et l'autre des systèmes de services seraient une barrière additionnelle à l'accès aux services. Toutefois, Woodward ne donne pas d'exemples de ces habitudes contraignantes.

La réponse au problème du manque d'intégration des services de santé et des services sociaux et communautaires est assez évidente : créer un contexte favorisant la collaboration, le travail d'équipe et la mise en commun des ressources. La question devient alors celle de comment créer un tel contexte. Une façon de procéder serait de tenter d'éliminer les barrières dont parle Woodward (1993). Par exemple, pour répondre au problème du cloisonnement professionnel et au problème d'attitude défavorable quant aux réalités associées au double diagnostic, il serait utile de mettre sur pied un programme conjoint de formation offert aux intervenants en santé et à ceux des services sociaux et communautaires. De tels programmes sont déjà disponibles, par exemple par l'entremise de National Association on Dual Diagnosis. À plus long terme, on pourrait espérer changer les cultures distinctes des programmes d'études médicales et de soins de santé d'une part, et d'études sociales et de services communautaires d'autre part, pour y inclure un volet «double diagnostic» commun aux deux types de programmes.

Une autre stratégie serait de souligner la complémentarité des pratiques existantes entre les intervenants en santé et ceux des services sociaux et communautaires. Il a été démontré que les déficits au plan des habiletés sociales sont associés à des troubles affectifs chez les personnes ayant un handicap intellectuel (Helsel et Matson 1988; Laman et Reiss 1987). Or, les intervenants des deux services travaillent au développement des habiletés sociales chez les personnes ayant un double diagnostic même s'ils poursuivent des objectifs différents. Les uns visent ainsi à faciliter tout le processus d'intégration sociale (Newton et Horner 1993) ou encore à développer un sentiment d'appartenance à la communauté (Walker 1999). Les autres visent à contrer les signes de troubles affectifs (par ex. la dépression). Il serait alors possible 
de créer des liens de travail entre les intervenants des différents services à partir de projets communs axés sur le développement durable d'habiletés sociales qui pourraient avoir une influence positive au plan psychologique.

\section{Conclusion}

Le présent article a décrit plusieurs obstacles à surmonter afin de pouvoir répondre adéquatement aux besoins en santé mentale des personnes ayant un double diagnostic. Il présente différents moyens pour améliorer la situation. En terminant,j'aimerais insister sur certains aspects.

D'abord, il est essentiel d'identifier clairement le nombre de personnes qui ont réellement un double diagnostic, afin de planifier adéquatement les services à leur offrir. Or, les estimés généraux faits à partir de recensements de la population ne peuvent être considérés sérieusement, surtout à la lumière des études cliniques et scientifiques qui ne parviennent pas à donner une image très précise de l'incidence des personnes ayant un double diagnostic. Ceci est particulièrement vrai pour les francophones de l'Ontario. Il est donc nécessaire de faire les études qui s'imposent pour clairement identifier leur nombre, leur milieu de vie et leurs besoins particuliers.

Ensuite, il est souhaitable de conserver la définition de double diagnostic telle que nous l'avons présentée ici et telle qu'elle apparaît dans les écrits cliniques et scientifiques, pour les raisons suivantes. Premièrement, elle facilite l'identification de personnes ayant de grands besoins. Deuxièmement, elle repose sur un corpus bien établi de recherches scientifiques de plus en plus nombreuses. Troisièmement, elle peut servir de point de ralliement pour ceux et celles qui travaillent au mieux-être psychologique des personnes qui ont un handicap intellectuel. Étendre la définition de double diagnostic aux personnes ayant des troubles spécifiques d'apprentissage, par exemple, serait maladroit et sans fondement scientifique ou empirique. 
Finalement, on doit encourager la recherche tant au plan social qu'au plan clinique (p. ex. sur les outils diagnostiques), de même que le travail de collaboration entre les systèmes de services en santé mentale et ceux des services sociaux et communautaires. Sur ce dernier point, la réussite pourrait dépendre non seulement de directives claires de la part des gestionnaires des services en cause, mais aussi (et peut-être surtout) de la mise sur pied de projets bien ciblés et pertinents pour les personnes qui en sont les récipiendaires, comme un projet de développement d'habiletés sociales en relation avec l'évaluation de son effet sur l'amélioration du mieux-être émotionnel.

\section{Bibliographie}

AMERICAN PSYCHIATRIC ASSOCIATION (1994). Diagnostic and Statistical Manual of Mental Disorders (4 ${ }^{\text {ième }}$ édition), Washington, D.C., American Psychiatric Association.

BAINE, D. (1996). Guide to Assessment and Instruction: Moderate and Severe Disabilities, Edmonton, Alberta,Vector.

BAROFF, G. S. (1996). «The Mentally Retarded Offender», dans J.W.Jacobson et J.A. Mulick (dir. de publ.), Manual of Diagnosis and Professional Practice in Mental Retardation, Washington, American PsychologicalAssociation, 311-322.

BLACK, L., C. CULLEN et R. W. NOVACO (1997). «Anger Assessment for People with Mild Mental Retardation in Secure Settings», dans B. Kroese, D. Dagnan, et K. Loumidis (sous la dir. de), Cognitive Therapy with People with Mental Retardation, London, Routlege.

BORTHWICK-DUFFY, S.A. et R. K.EYMAN (1990). «Who are the Dually Diagnosed ?», American Journal of Mental Retardation, no 94, 586-595.

BRUININKS, R. H.., B.K. HILL et L.E. MORREAU (1988). «Prevalence and Implications of Maladaptive Behaviors and Dual Diagnosis in Residential and Other Service Programs», dans J. A. Stark, F. J. Menolascino, M. H.. Albarelli, et V. C. Gray (sous la dir. de), Mental Retardation and Mental Health : Classification, Diagnosis, Treatment, Services, New York, Springer-Verlag, 3-29

CHENG, L .L. (1987). Assessing Asian Language Performance : Guidelines for Evaluating Limited-EnglishProficient Students, Rockville, Aspen Publishers.

DAS, J. P. et J.A. NAGLIERI (1996). «Mental Retardation and Assessment of Cognitive Processes», dans J.W. Jacobson et J. A. Mulick (sous la dir. de), Manual of Diagnosis and Professional Practice in Mental Retardation, Washington, American Psychological Association, 115-126.

DOSËN,A. (1993). «Diagnosis and Treatment of Psychiatric and Behavioural Disorders in Mentally Retarded Individuals : The State of The Art», Journal of Intellectual Disability Research, no 37, 1-7.

GARNER,W. I., et C. L. COLE (1993). «Aggression and Related Conduct Disorders : Definition, Assessment, and Treatment», dans Matson, J. L., et Barrett, R. P. (sous la dir. de), Psychopathology in The Mentally Retarded, Boston, Allyn and Bacon, 213-252. 
GARDNER,W. I. et C.W. MOFFAT (1990). "Aggressive Behavior : Definition, Assessment, Treatment», International Review of Psychiatry, no 2, 91-100.

HARDAN, A. et R. SAHL (1999). «Suicidal Behavior in Children and Adolescents With Developmental Disorders», Research in Developmental Disabilities, no 20, 287-296.

HELSEL,W.J. et J.L. MATSON (1988). «The Relationship of Depression to Social Skills and Intellectual Functioning in Mentally Retarded Adults", Journal of Mental Deficiency Research, vol. 32, 411-418.

JACOBSON,J.W. (1990). «Do Some Mental Disorders Occur Less Frequently Among Persons with Mental Retardation?», American Journal on Mental Retardation, vol. 94, 596-602.

LAMAN, D. S. et S. REISS (1987). «Social Skill Deficiencies Associated With Depressed Mood of Mentally Retarded Adults», American Journal of Mental Deficiency, vol. 92, 224-229.

LANGER, E. J. et R.P. ABELSON (1974). «A Patient By Any Other Name : Clinician Group Differences in Labeling Bias", Journal of Consulting and Clinical Psychology, vol. 42, 4-9.

LIM, H. F. L. et D.M. BROWDER (1994). «Multicultural Life Skills Assessment of Individuals with Severe Disabilities», The Journal of The Association for Persons with Severe Handicaps, no 19,130-138.

LUCKASSON, R., D.L.COULTER, E.A.POLLOWAY, S. REISS, R.L. SCHALOCK, M.E. SNELL, D.M.SPITALNIK et J.A.STARK (1992). Mental Retardation: Definition, Classification, and Systems of Supports, Washington, D.C., Mental retardation:American Association on Mental Retardation.

MACLEAN,W.E. (1993). «Overview», dans Matson,J.L., et R.P. Barrett (sous la dir. de), Psychopathology in The Mentally Retarded, Boston, Allyn and Bacon, 1-16.

MARTIN, N. T., E.A. GAFFAN et T. WILLIANS (1999). «Experimental Functional Analyses for Challenging Behavior : A Study ofValidity and Reliability», Research in Developmental Disabilities, no 20, 125-146.

MATSON, J. L., J.W. BAMBURG, K.E. CHERRY et T.E PACLAWSKYJ (1999). «A Validity Study on the Questions About Behavioral Function (QABF) Scale : Predicting Treatment Success for Self-Injury, Aggression, and Stereotypes», Research in Developmental Disabilities, no 20,163-176.

MATSON, J. L. et R.P. BARRETT (1993). Psychopathology in the Mentally Retarded. Boston, Allyn and Bacon.

MATSON,J.L., K.S.RUSH, M.HAMILTON, S.J.ANDERSON,J.W.BAMBURG et C.S. BABLIO (1999). "Characteristics of Depression as Assessed by the Diagnostic Assessment for the Severely Handicapped-II (DASH-II)», Research in Developmental Disabilities, no 20, 305-313.

MATSON, J. L. et J.A. SEVIN (1994). «Theories of Dual Diagnosis in Mental Retardation», Journal of Consulting and Clinical Psychology, no 62, 6-16.

NEWTON, J. S. et R.H. HORNER (1993). «Using a Social Guide to Improve social relationships of people with severe disabilities», Journal of the Association for Persons with Severe Handicaps, vol. $18,36-45$.

NEZU, C. M., et A.M. NEZU (1994). «Outpatient Psychotherapy for Adults with Mental Retardation and Concomitant Psychopathology : Research and Clinical Imperatives», Journal of Consulting and Clinical Psychology, no 62, 34-42.

NEZU, C. M., A.M. NEZU et M.J. GILL-WEISS (1992). Psychopathology in Persons with Mental Retardation: Clinical Guidelines for Assessment and Treatment. Champaign, IL, Research Press.

PAWLARCZYCK, D. et B.E. BECKWITH (1987). «Depressive Symptoms Displayed by Persons with Mental Retardation : A Review», Mental Retardation, no 25, 325-330. 
REISS, S. (1990). «Prevalence of Dual Diagnosis in Community-Based Day Programs in the Chicago Metropolitan Area", American Journal of Mental Retardation, no 94, 578-585.

REISS, S. (1993). «Assessment of Psychopathology in Persons with Mental Retardation», dans J.L. Matson et R.P. Barrett (sous la dir. de), Psychopathology in the Mentally Retarded, Boston, Allyn and Bacon, 17-40

Reiss, S. (1994). Handbook of Challenging Behavior : Mental Health Aspects of Mental Retardation, Worthington, OH, International Diagnostic Systems.

REISS, S., G.W. LEVITAN et R.J. MCNALLY (1982). «Emotionally Disturbed Mentally Retarded People : An Underserved Population", American Psychologist, vol. 37, 361-367.

REISS, S., G.W.LEVITAN et J.SZYSZKO (1982). «Emotional Disturbance and Mental Retardation : Diagnostic Overshadowing», American Journal of Mental Deficiency, no 86. 567-574.

REISS, S. et J. SZYSZKO (1983). «Diagnostic Overshadowing and Professional Experience with Mentally Retarded Persons», American Journal of Mental Deficiency, no 87, 396-402.

REISS, S. et D. VALENTI-HEIN (1994). «Development of a Psychopathology Rating Scale for Children with Mental Retardation", Journal of Consulting and Clinical Psychology, no 62, 28-33.

ROJHAN, J. et M.J.TASSÉ (1996). «Psychopathology in Mental Retardation», dans J.W. Jacobson et J. A. Mulick (sous la dir. de), Manual of Diagnosis and Professional Practice in Mental Retardation, Washington, American Psychological Association, 147-156

SMITH, J. D. (1994). «The Revised AAMR Definition of Mental Retardation : the MRDD Position», Education and Training in Mental Retardation and Developmental Disabilities, September, 179-183.

SINGH, N. N., A. SOOD, N. SONENKLAR et C.R. ELLIS. (1991). "Assessment and Diagnosis of Mental Illness in Persons with Mental Retardation", Behavior Modification, vol. 15, 419-443.

SOVNER, R. et R.J. PARY (1993). "Affective Disorders in Developmentally Disabled Persons», dans J.L. Matson, et R.P. Barrett (sous la dir. de), Psychopathology in The Mentally Retarded, Boston, Allyn and Bacon, 87-147

SURMEY,P., M. REED et J. CORBETT (1991). «Psychometric Assessment of Psychiatric Disorders in People with Learning Difficulties (mental handicap) : A Review of measures», Psychological Medicine, VOL. 21, 143-155.

TANGYAY, P. E. et L.S. SZYMANSKI (1980). «Training of Mental Health Professionals in Mental Retardation", dans L. S. Szymanski et P. E.Tanguay (sous la dir. de), Emotional Disorders of Mentally Retarded Persons : Assessment, Treatment, and Consultation, Baltimore, University Park Press, 19-28 VAN HOUTEN, R. et S.AXELROD (1993). Behavior Analysis and Treatment. NewYork, Plenum Press. WALKER, P. (1999). «From Community Presence to Sense of Place : Community Experiences of Adults with Developmental Disabilities», Journal of the Association for Persons with Severe Handicaps, no 24, 23-32.

WALSH, K. K. et N. SHENOUDA (1999). "Correlations Among the Reiss Screen, the Adaptive Behavior Scale Part II, and the Aberrant Behavior Checklist», American Journal on Mental Retardation, no 104, 236-248.

WEISZ, J. R. (1981). «Effect of the «Mentally Retarded» Label on Adult Judgments about Child Failure», Journal of Abnormal Psychology, no 90,371-374.

WOODWARD, H. L. (1993). «One Community's Response to the Multi-system Service Needs of Individuals with Mental Illness and Developmental Disabilities», Community Mental Health Journal, no 29, 347-359. 


\section{Notes}

1. Dans la suite du texte, on utilisera l'expression «déficience mentale» comme étant synonyme.

2. Pour une discussion détaillée de ce phénomène, voir Maclean (1993).

3. Pour une discussion détaillée du lien entre la démarche diagnostique de la déficience mentale et les facteurs sur lesquels elle repose, voir Luckasson, Coulter, Polloway, Reiss, Schalock, Snell, Spitalnik et Stark (1992).

4. Pour un résumé de ces outils et des stratégies d'évaluation, voir Reiss (1993) et Rojahn et Tassé (1996). 